\title{
Band-Edge Lasing in Gold-Clad Photonic-Crystal Membranes
}

\author{
Joris Van Campenhout, Student Member, IEEE, Peter Bienstman, Member, IEEE, and Roel Baets, Senior Member, IEEE
}

\begin{abstract}
We investigate the possibility to achieve band-edge lasing in optically thick gold-clad photonic-crystal (PhC) membranes, with a dielectric thickness of around $1 \mu \mathrm{m}$. We have performed a two-dimensional eigenmode-expansion analysis of band-edge resonators in one-dimensional PhCs. Material thresholds, quality factors, and emission efficiencies have been calculated for TE band-edge laser resonances on the second and third $\Gamma$-point. The second $\Gamma$-point sustains band-edge laser modes with quality factors above 2500 for a membrane thickness of 1 $\mu \mathrm{m}$ and a cavity length of 20 periods, however, with a very poor surface-emission efficiency. Band-edge laser modes located on the third $\Gamma$-point have lower quality factors but higher surface-emission efficiencies. In both cases, the $\mathrm{PhC}$ should be designed specifically to avoid coupling with lossy, higher order modes.
\end{abstract}

Index Terms-Eigenmode expansion, metallic waveguides, microlasers, photonic crystals (PhCs).

\section{INTRODUCTION}

$\mathbf{P}$ HOTONIC crystals (PhCs) have lately attracted a lot of interest due to their ability to control light on a micron-sized scale. A lot of functionality can be achieved using the mirror effect due to the photonic bandgap, e.g., in waveguides. Other applications are based on the reduction of group velocity near the photonic band edges, for example, in lasing action or enhancement of nonlinear phenomena.

$\mathrm{PhC}$ lasers can basically be classified in defect-based lasers and defect-free lasers. The former relies on the mirror effect of the $\mathrm{PhC}$ around a carefully designed defect that forms a cavity with high-quality factors and small mode volumes [1]. The latter is based on the gain enhancement effect [2]-[4] near the band edges and the increased bloch-reflectivity at the PhC-bulk interface, in the case of finite-size band-edge lasers. Mode volumes of band-edge lasers are typically somewhat larger.

Band-edge lasers based on two-dimensional (2-D) $\mathrm{PhC}$ membranes have lately both been fabricated [5]-[7] and analyzed numerically [8]. However, all these efforts included optically pumped devices. For practical devices, electrical pumping will be essential. Electrically pumped band-edge lasing in a 2-D PhC has been demonstrated by Noda et al. [9]. However, this device was based on a low-contrast grating structure, so the device has a diameter of more than $100 \mu \mathrm{m}$.

Manuscript received November 4, 2004. The work of J. Van Campenhout and P. Bienstman were supported in part by the Flemish Fund for Scientific Research (FWO-Vlaanderen) through a doctoral fellowship and postdoctoral fellowship, respectively.

The authors are with the Photonics Research Group, Department of Information Technology (INTEC), Ghent University-IMEC, Ghent B-9000, Belgium (e-mail: Joris.VanCampenhout@intec.UGent.be).

Digital Object Identifier 10.1109/JSAC.2005.851160
In this paper, we demonstrate theoretically that it is possible to achieve surface-emitting band-edge lasing in a membranetype device with a very simple electrical contacting scheme, i.e., by putting one contact on top and one on the bottom of the membrane. The cavity consists of a gold-clad PhC membrane with a dielectric thickness of around $1 \mu \mathrm{m}$ (optically thick). The gold layers act as highly reflective electrical contacts, a combination that can be achieved with nonalloyed Au contacts [10]. Cavity diameters can go down to less than $30 \mu \mathrm{m}$. This band-edge laser can be useful for integration purposes of electronic and photonic functions, for which there can be a need for tradeoff between emitted power and compactness. The band-edge resonators are very suitable to cover this scope. Compared with VCSELs, these devices have a reduced thickness and promise an improved modal stability.

We have performed a numerical, 2-D analysis of these gold-clad $\mathrm{PhC}$ cavities using the eigenmode expansion technique [11]. Rather than working with a grid, the structure is divided in a number of layers, where the refractive index profile does not change in the $z$ direction. The fields in each layer are written as a sum of the local eigenmodes of that particular layer. Scattering matrices are calculated at each interface. The computational domain has absorbing boundaries to prevent any interference with reflected waves. To calculate laser modes, the cavity-containing some active material with a complex refractive index - is divided into two parts, for which the reflection matrices $\mathbf{R}_{\text {left }}$ and $\mathbf{R}_{\text {right }}$ are calculated. A laser mode $\mathbf{A}_{\text {las }}$ then has to satisfy the resonance condition

$$
\mathbf{R}_{\text {left }} \mathbf{R}_{\text {right }} \mathbf{A}_{\text {las }}=\mathbf{A}_{\text {las }} .
$$

To fulfill this condition, the wavelength and imaginary part of the refractive index of the active material are adjusted. These calculations yield the resonance wavelength and the material threshold gain, as well as the field profile of the resonant mode. The used modeling tool CAMFR is freely available from [12].

The rest of this paper is organized as follows. In Section II, we perform a modal analysis of a nonpatterned optically thick gold-clad waveguide, as a function of the dielectric thickness. The patterning is added in Section III to form a photonic-crystal waveguide (PhC-WG). In Section IV, band-edge resonators based on the PhC waveguide of Section III are analyzed. Finally, a conclusion is drawn in Section VI.

\section{Gold-Clad DieleCtric WAVEGUide}

Consider the layer structure depicted in Fig. 1: a symmetric dielectric waveguide consisting of cladding layers with thickness $t_{1}$ and confinement layers with thickness $t_{2}$. Four 


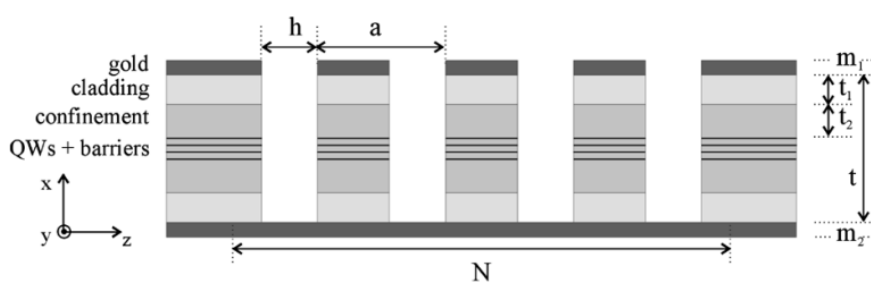

Fig. 1. Layer structure of a gold-clad PhC-WG.

TABLE I

REFRACTIVE INDICES OF GOLD-CLAD DIELECTRIC WAVEGUIDE

\begin{tabular}{l|l|l}
\hline Material & Refractive index & Thickness \\
\hline Gold & $0.54-9.6 \mathrm{j}$ & $m_{1}, m_{2}$ \\
\hline Cladding layer & 3.17 & $t_{1}$ \\
\hline Confinement layer & 3.38 & $t_{2}$ \\
\hline Barrier layer & 3.38 & $20 \mathrm{~nm}$ \\
\hline Quantum well & 3.40 & $5 \mathrm{~nm}$ \\
\hline
\end{tabular}

5-nm-thick quantum wells are embedded in 20-nm-thick barrier layers. This dielectric waveguide is clad with thick gold layers on both sides. The refractive indexes of the used materials are listed in Table I. Gold was chosen as cladding metal due to the low absorption loss in the infrared region as compared with other-nonnoble-metals. In this section, the quantum wells are assumed to be transparent, and the waveguide is not patterned, i.e., the waveguide is a one-dimensional (1-D) slab.

Modal absorption losses at an operating wavelength of $1.5 \mu \mathrm{m}$ are depicted as a function of total dielectric thickness $t$ in Fig. 2, both for TE-(electric field in the $y$ direction) as TM-polarization. For each thickness $t, t_{1}$, and $t_{2}$ have been chosen to yield the lowest modal absorption loss for the fundamental $\mathrm{TE}_{0}$-mode. The waveguide structure is monomode for $t<0.45 \mu \mathrm{m}$. However, in this range, $\mathrm{TE}_{0}$ absorption losses are too high to use the waveguide in a laser resonator. For $0.45 \mu \mathrm{m}$, the waveguide becomes multimode and the absorption loss of modes far above cutoff decreases almost exponentially as a function of $t$, as progressively less of the field penetrates into the lossy gold layer. For TM, absorption losses are more than one order of magnitude higher than for the fundamental $\mathrm{TE}_{0}$-mode. For $t$ around $1 \mu \mathrm{m}$, the propagation loss of the $\mathrm{TE}_{0}$-mode is around $15 \mathrm{~cm}^{-1}$. For the remainder of this paper, we will assume TE-polarization and an operating wavelength around $1.5 \mu \mathrm{m}$.

\section{Gold-Clad Photonic-Crystal WAVEGUIDE (PhC-WG)}

Suppose that the waveguide structure of Fig. 1 is patterned with rectangular holes of width $h$ and period $a$. We assume that the bottom gold layer remains unpatterned. This way we have created an optically thick, gold-clad 1-D PhC-WG.

Fig. 3 shows the band diagram for $a=665 \mathrm{~nm}$ and $h=$ $210 \mathrm{~nm}$. Although not a limitation of the model, the dispersive character of the refractive index of gold has been ignored in this calculation and, therefore, the result is only physical around $1.5 \mu \mathrm{m}$ or for reduced frequencies $\Omega=a / \lambda$ around 0.443 . Still, this approximate band structure gives a good qualitative view of the modal properties of the gold-clad PhC-WG. The bands

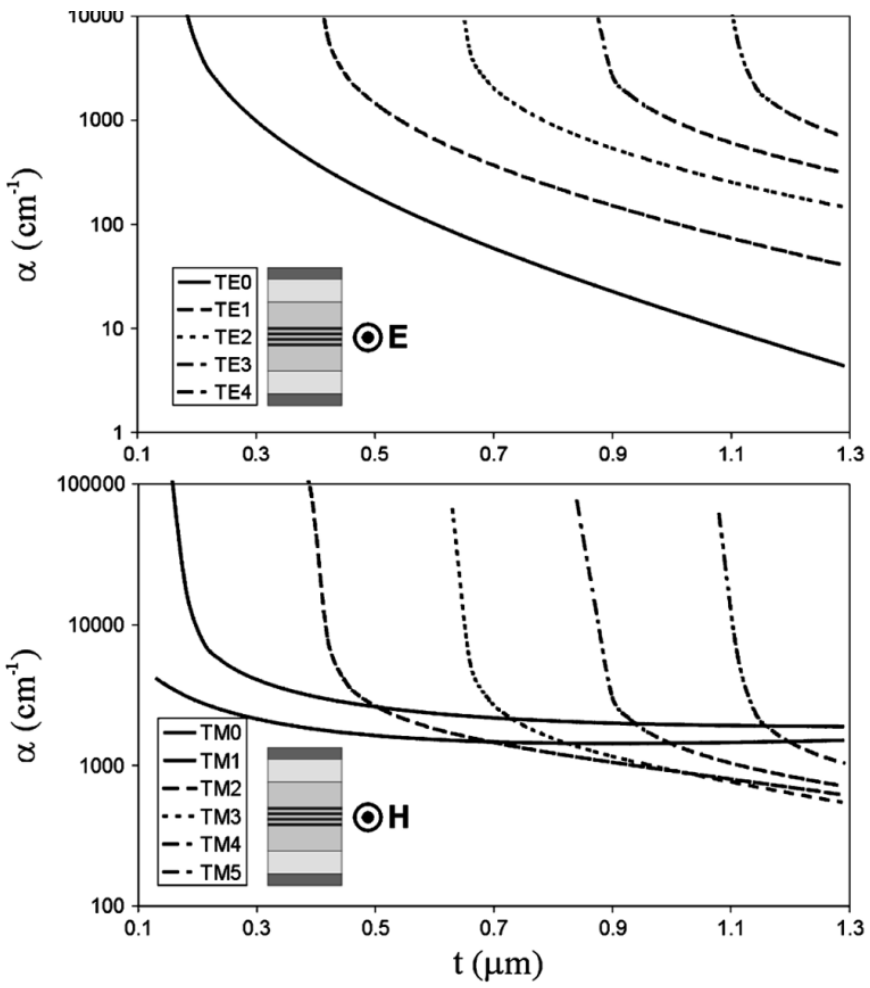

Fig. 2. Modal absorption losses of the fundamental and higher order modes of the waveguide structure as depicted in Fig. 1 (without patterning), at $1.5 \mu \mathrm{m}$. The $\mathrm{TM}_{0}$ and $\mathrm{TM}_{1}$ modes are plasmon-like.

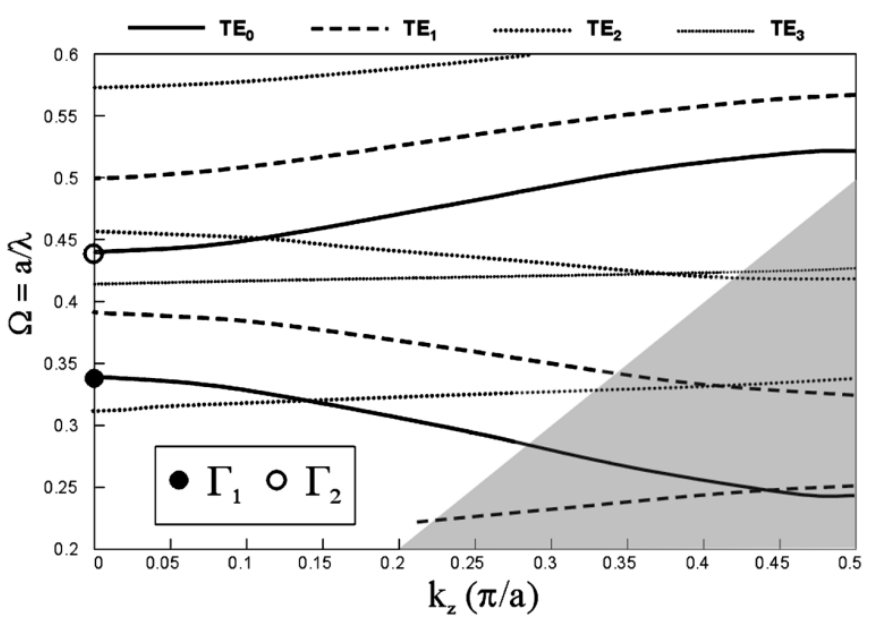

Fig. 3. TE band structure of the gold-clad PhC-WG with $a=665 \mathrm{~nm}, h=$ $210 \mathrm{~nm}, t=1000 \mathrm{~nm}$, and $m_{1,2}=100 \mathrm{~nm}$. The region below the light line is in gray. The MSBs are not visible on the scale of the figure.

of the fundamental and the higher order modes are folded back due to the periodicity and bandgaps are opened near the edges and the center of the Brillouin zone. At the same time, ministopbands (MSB) are formed where two bands cross. Since there is no vertical mirror symmetry and, hence, no strict $x$-even- $x$-odd classification, a MSB can open regardless of the order of the two interacting modes. Due to their limited size, the MSBs are not visible in Fig. 3.

Modes above the light line (LL) can couple to radiation modes, through the patterned top gold layer. However, due to symmetry mismatch, some modes couple only very weakly to radiation modes, even above the LL [13]. The $\mathrm{TE}_{0}$-mode 


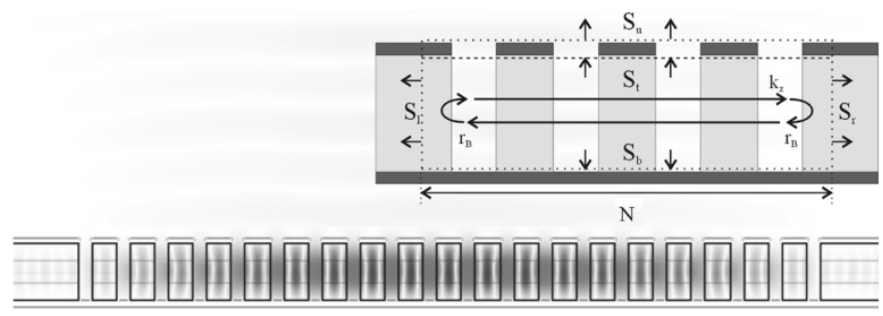

Fig. 4. $\quad \Gamma_{2}$ band-edge laser mode (E-field). The inset shows the definition of power fluxes.

is antisymmetric with respect to $S_{z}$ (mirror symmetry around $z=0)$ at $\Gamma_{1}$, whereas it is symmetric under $S_{z}$ at $\Gamma_{2}$. A plane wave $\exp \left(-j k_{0} x\right)$ propagating in the $x$ direction is symmetric under $S_{z}$, thus, the $\mathrm{TE}_{0}$-mode cannot couple to radiation modes at $\Gamma_{1}$, whereas it is leaky at $\Gamma_{2}$.

\section{BAND-EDGE LASING}

\section{A. Band-Edge Lasing Principle}

Consider the band-edge resonator that is shown in Fig. 4. Resonant modes with a high-quality factor $Q$ can be built up for frequencies near the edges of the photonic bands, where the group velocity $v_{g}=d \omega / d k$ is very low. A Bloch-mode propagating in an active $\mathrm{PhC}$ encounters a gain per unit length that is enhanced with a factor inversely proportional to $v_{g}$, as compared with a properly normalized bulk gain [4]. Moreover, the amplitude of the field reflection $r_{B}$ of the Bloch-mode at the edges of the finite $\mathrm{PhC}$ cavity increases when approaching a band edge. The resonance condition can be written in good approximation as

$$
r_{B}^{2} e^{-j k_{z} 2 L}=1
$$

in which $k_{z}$ is the complex propagation constant of the Bloch mode and $L=N a$. The amplitude condition of this equation fixes the threshold gain, and the phase condition determines the resonance wavelength. The phase condition can be fulfilled for several longitudinal modes. In what follows, we will only consider the longitudinal mode which lies closest to the band edge and, hence, has the lowest threshold gain.

As indicated in Section III, there are two types of $\mathrm{TE}_{0}$-modes: decoupled modes that exhibit little or no vertical radiation loss, e.g., near $\Gamma_{1}$, and leaky modes that have a larger vertical radiation loss, e.g., near $\Gamma_{2}$. We searched for band-edge laser modes for both types of $\mathrm{TE}_{0}$-modes.

\section{B. Band-Edge Lasing at $\Gamma_{1}$ (Decoupled TE $E_{0}$-Mode)}

If we set $a=560 \mathrm{~nm}, h=260 \mathrm{~nm}, t=1.0 \mu \mathrm{m}$, and $m_{1,2}=100 \mathrm{~nm}$, the $\Gamma_{1}$-point lies at a reduced frequency of $\Omega=0.377$ or $\lambda=1.485 \mu \mathrm{m}$. We used this gold-clad PhC-WG to form a band-edge resonator with $N$ periods (see Fig. 4). Then, we searched for laser modes near the $\Gamma_{1}$-point. In these calculations, the wavelength and the material gain in the quantum wells are adjusted to fulfill the lasing condition. Each lasing mode is then characterized by a resonance wavelength $\lambda_{r}$ and threshold material gain $G_{\text {th }}$. We have also calculated the $Q$-factor of the lasing modes, which is given by

$$
Q=\omega_{r} \frac{U_{\text {stored }}}{S}
$$

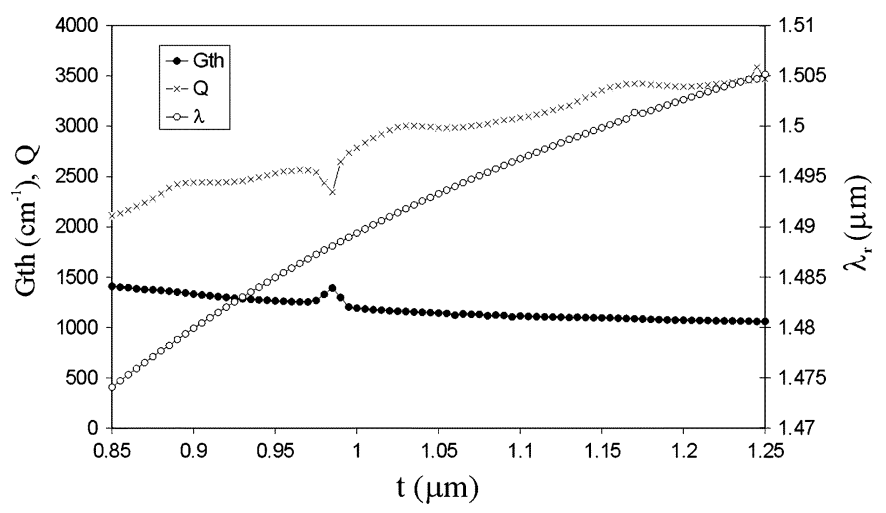

Fig. 5. Properties $G_{\text {th }}, \lambda_{r}$, and $Q$-factor of the $\Gamma_{1}$ band-edge laser mode as a function of membrane thickness $t$, with $a=560 \mathrm{~nm}, h=260 \mathrm{~nm}, m_{1,2}=$ $100 \mathrm{~nm}$, and $N=20$.

where $U_{\text {stored }}$ is the energy stored in the resonator, $\omega_{r}$ is the resonance frequency, and $S$ is defined as the power flux leaving the cavity. $S$ can be decomposed as follows:

$$
S=S_{b}+S_{t}+S_{l}+S_{r}
$$

$S_{b}, S_{t}, S_{l}$, and $S_{r}$ are, respectively, defined as the power flux through the bottom, top, left side, and right side of the cavity (see Fig. 4). Due to the mirror symmetry of the cavity, $S_{l}$ is equal to $S_{r}$ and we define $S_{\text {side }}=S_{r}+S_{l}$. We also define $S_{u}$ as the total power flux leaving the cavity above the top gold layer. $S_{u}$ can be considered as the useful extracted power flux, and $S_{u} / S$ as the emission efficiency. $S_{m}=S_{b}+S_{t}-S_{u}$ can then be considered as the power flux lost in the gold layers.

An alternative definition of the $Q$-factor of the resonant mode is given by

$$
Q=\frac{\omega_{r}}{\Delta \omega}
$$

where $\Delta \omega$ is the resonance's full linewidth at half maximum. We derived $\Delta \omega$ by inspecting the $\left|\mathbf{E}^{2}\right|$-spectrum (at a point in the cavity) that is obtained by performing a wavelength sweep for a well-placed dipole source in the band-edge resonator, with transparent quantum wells. The resulting $Q$-values were comparable with the ones obtained with (3).

The results for $N=20$ near $\Gamma_{1}$ are depicted as a function of $t$ in Fig. 5. The resonance wavelength $\lambda_{r}$ is close to the $\Gamma_{1}$ band-edge, and increases monotonically with increasing $t$, since the effective index of the fundamental $\mathrm{TE}_{0}$ increases with increasing $t . G_{\text {th }}$ decreases from $1410 \mathrm{~cm}^{-1}$ for $t=0.85 \mu \mathrm{m}$ to $1060 \mathrm{~cm}^{-1}$ for $t=1.25 \mu \mathrm{m}$. The $Q$-factor increases from 2100 to 3500 . This is due to the reduced absorption loss for thicker membranes and, to a lesser extent, the reduced radiation loss. There is a peak apparent for $t=0.985 \mu \mathrm{m}$ both in the $G_{\mathrm{th}}(+15 \%)$ and the $Q$-curve $(-10 \%)$. The interpretation of this peak will be given in IV.D. The required $G_{\mathrm{th}}$-values are well within range of optical gain levels in compressively strained quantum wells [14].

Calculation of the power fluxes indicates that $S_{u}$ accounts for only less than $2 \%$ of the total power loss. For $t=0.85 \mu \mathrm{m}$, $S_{m} / S$ is almost $62 \%$, and $S_{\text {side }} / S$ is $37 \%$. For $t=1.25 \mu \mathrm{m}$, this is $S_{m} / S=24 \%$ and $S_{\text {side }} / S=75 \%$. The low $S_{u}$-values can be 


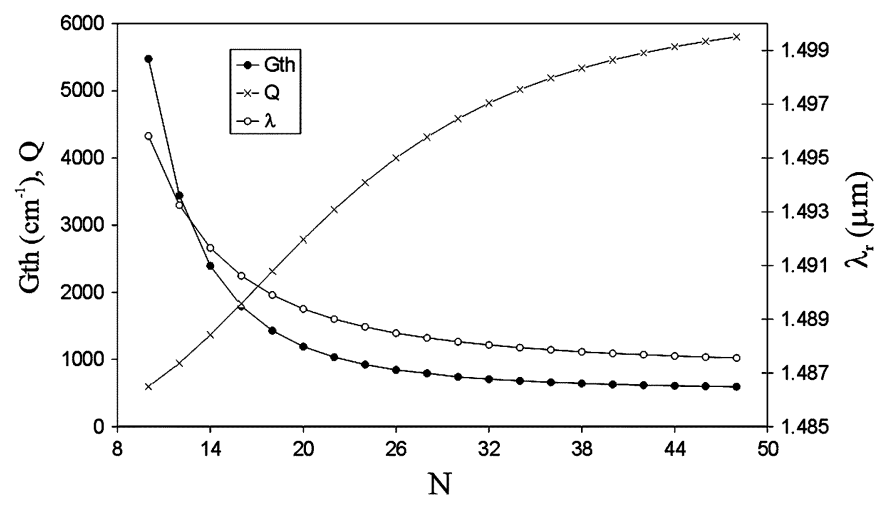

Fig. 6. Properties $G_{\text {th }}, \lambda_{r}$, and $Q$-factor of the $\Gamma_{1}$ band-edge laser mode as a function of number of periods $N$, with $a=560 \mathrm{~nm}, h=260 \mathrm{~nm}, m_{1,2}=$ $100 \mathrm{~nm}$, and $t=1.0 \mu \mathrm{m}$.

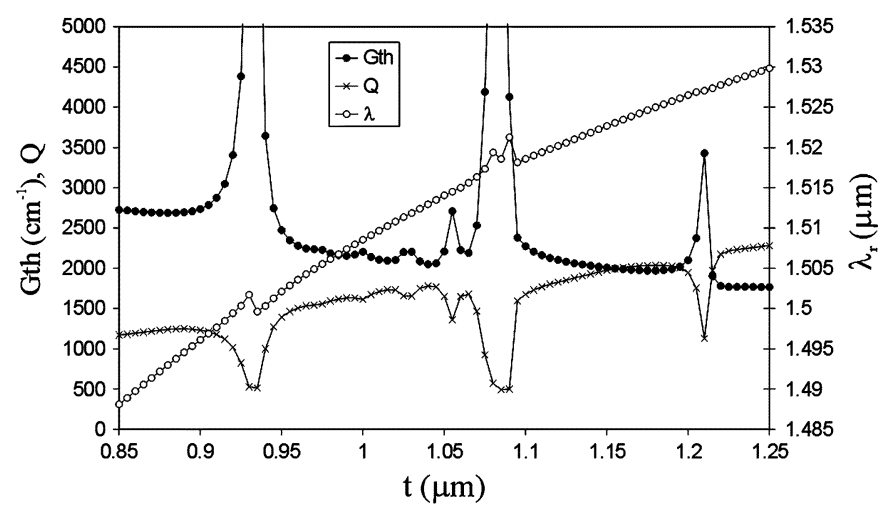

Fig. 7. Properties $G_{\mathrm{th}}, \lambda_{r}$, and $Q$-factor of the $\Gamma_{2}$ band-edge laser mode as a function of membrane thickness $t$, for $a=665 \mathrm{~nm}, h=210 \mathrm{~nm}, m_{1,2}=$ $100 \mathrm{~nm}$, and $N=20$.

understood in terms of the nonleaky character of the $\mathrm{TE}_{0}$-mode near $\Gamma_{1}$.

Finally, the evolution of $G_{\mathrm{th}}, Q$, and $\lambda_{r}$ for $t=1.0 \mu \mathrm{m}$, as a function of the number of periods $N$, is shown in Fig. 6. The resonance wavelength approaches the band edge for progressively longer cavities, as can be understood from the phase condition of (2). For $N=40, G_{\mathrm{th}}$ is as low as $630 \mathrm{~cm}^{-1}$, with a $Q$ of 5460. The total threshold power $G_{\mathrm{tot}}=G_{\mathrm{th}} L=G_{\mathrm{th}} \mathrm{Na}$ is minimal for $N=26$.

\section{Band-Edge Lasing at $\Gamma_{2}$ (Leaky $T E_{0}$-Mode)}

If we set $a=665 \mathrm{~nm}, h=210 \mathrm{~nm}, t=1000 \mathrm{~nm}$, and $m_{1,2}=100 \mathrm{~nm}$, the $\Gamma_{2}$-point lies at a reduced frequency of $\Omega=0.44$ or $\lambda=1.511 \mu \mathrm{m}$. Again, we searched for lasing modes around $\Gamma_{2}$. The results for $N=20$ as a function of $t$ can be found in Fig. 7. Generally, threshold values $G_{\text {th }}$ are about two times higher and $Q$-values two times smaller as compared to the $\Gamma_{1}$-case. Furthermore, three distinct peaks are apparent both in the $G_{\mathrm{th}}$-curve as in the $Q$-curve: they appear for $t=0.93 \mu \mathrm{m}$, $t=1.08 \mu \mathrm{m}$, and $t=1.21 \mu \mathrm{m}$, respectively. Compared with the $\Gamma_{1}$-case, they are much more pronounced: $G_{\mathrm{th}}$-values increase with more than a factor 3 , whereas $Q$-values drop with the same factor. Interpretation of this feature will be given in IV.D.

The $S_{u}$-values are somewhat bigger compared with the $\Gamma_{1}$-case, due to the leaky character of the $\mathrm{TE}_{0}$-mode near $\Gamma_{2}$ : for $t=1.0 \mu \mathrm{m}$, we find $S_{u} / S=12.5 \%$, with $S_{m} / S=53 \%$

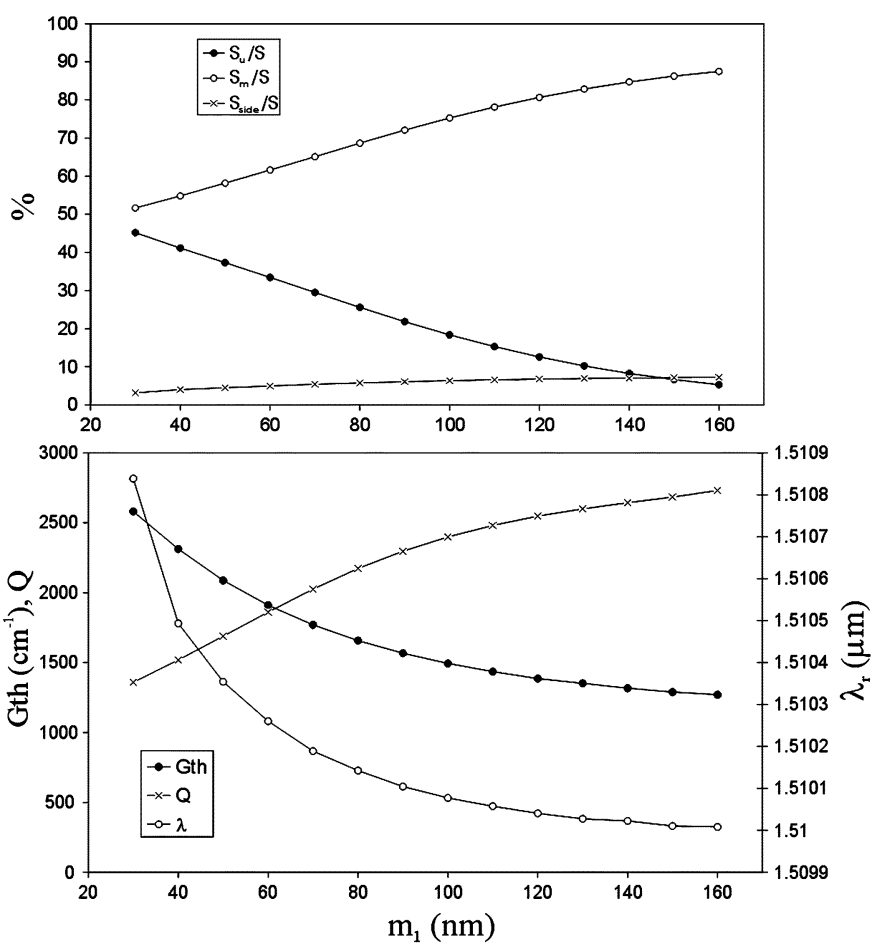

Fig. 8. $\quad \Gamma_{2}$ laser mode properties $G_{\mathrm{th}}, Q, \lambda_{r}, S_{u} / S, S_{m} / S$, and $S_{\text {side }} / S$, as a function of the top gold layer thickness $m_{1}$, for $a=665 \mathrm{~nm}, h=210 \mathrm{~nm}$, $t=1.0 \mu \mathrm{m}, m_{2}=100 \mathrm{~nm}$, and $N=40$.

and $S_{\text {side }} / S=34.5 \%$, for $G_{\text {th }}=2206 \mathrm{~cm}^{-1}$. The $S_{u} / S$-value can be increased by decreasing the thickness of the top gold layer $m_{1}$. However, this implies an increase in threshold material gain $G_{\mathrm{th}}$. As in the $\Gamma_{1}$-case, this can be countered by choosing a longer cavity size $L=N a$, at the price of an increased total threshold power $G_{\text {tot }}$. The results for $N=40$ are shown in Fig. 8, as a function of $m_{1}$. For $m_{1}=60 \mathrm{~nm}$, we find $S_{u} / S=33.5 \%, S_{m} / S=61.5 \%, S_{\text {side }} / S=5 \%$ for $G_{\mathrm{th}}=1910 \mathrm{~cm}^{-1}$ and a $Q$ of 1860 .

\section{Interpretation of the Peaks in the $G_{\mathrm{th}^{-}}$-and Q-Curves}

Both for $\Gamma_{1}$ as for $\Gamma_{2}$ band-edge laser modes, $G_{\mathrm{th}}{ }^{-}$and $Q$-values peak at specific membrane thicknesses. In order to interpret these results, we calculated band diagrams for these specific gold-clad PhC-WGs. For instance, Fig. 9 shows a detail near $\Gamma_{2}$ of the band diagram for $t=1.07 \mu \mathrm{m}$. A band extreme of the $\mathrm{TE}_{2}$-mode is located near the $\Gamma_{2}$-point of the fundamental $\mathrm{TE}_{0}$-mode. $\mathrm{A} \mathrm{MSB}$ is opened up due to the coupling between these two modes. Under these conditions, there is a power loss from the $\mathrm{TE}_{0}$-mode to the very lossy $\mathrm{TE}_{2}$-mode, which accounts for the increased threshold gain and the reduced $Q$-factor. For $t=0.93 \mu \mathrm{m}$ and $t=1.21 \mu \mathrm{m}$, there is an analogue coupling to the $\mathrm{TE}_{3}$-mode and the $\mathrm{TE}_{4}$-mode, respectively. The same goes for the $\Gamma_{1}$-case. For instance, a $\mathrm{TE}_{3}$-mode is apparent near $\Gamma_{1}$ for $t=0.985 \mu \mathrm{m}$. However, the peak is less pronounced, indicating a less effective coupling.

\section{FUTURE EXPERIMENTAL DEMONSTRATION}

Experimental demonstration of the concept of band-edge lasing in gold-clad $\mathrm{PhC}$ membranes can be realized in 2-D 


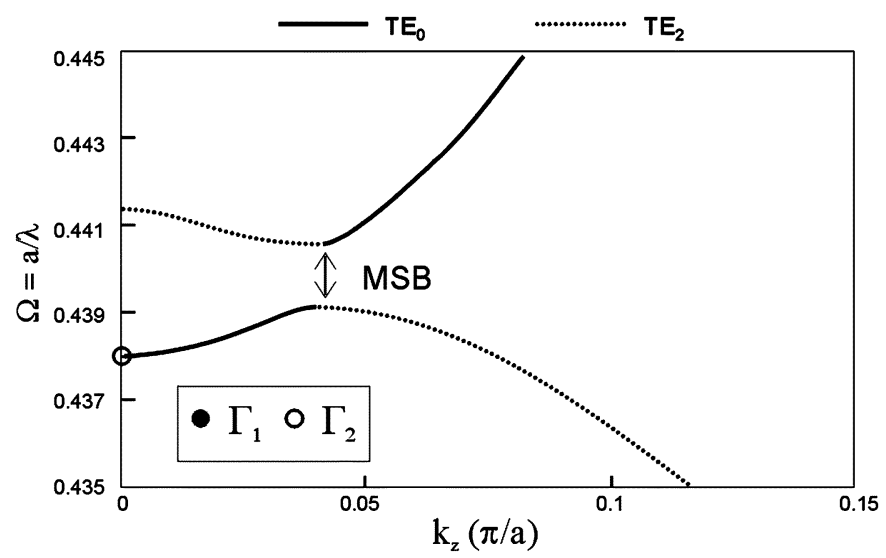

Fig. 9. Detail of the band diagram near $\Gamma_{2}$ for a gold-clad PhC-WG with $t=$ $1.07 \mu \mathrm{m}$, for $a=665 \mathrm{~nm}, h=210 \mathrm{~nm}$, and $m_{1,2}=100 \mathrm{~nm}$. A MSB is opened up due to coupling between the $\mathrm{TE}_{0}$ - and $\mathrm{TE}_{2}$-mode.

PhC cavities. The design of the device then includes the band structure calculation of metallized 2-D PhCs, so to avoid coupling of the fundamental $\mathrm{TE}_{0}$-mode with higher order modes. However, in the 2-D case, one should also avoid coupling with higher order TM-like modes, so a specific design is essential. The fabrication of the device could be done in the following manner. First, the bottom Au contact is deposited on a specifically grown epi-structure. Then, this metallized epi-structure is bonded to a carrier with a low temperature bonding process, for instance, BCB-bonding [15]. High-temperature bonding is not allowed to avoid alloying of the Au-contact, since this increases absorption losses. Subsequently, the substrate of the epi-structure is removed and the $\mathrm{PhC}$ pattern is etched in the resulting membrane. Finally, the top Au contact is deposited by oblique evaporation, for which a shadowing effect avoids filling up the PhC holes. First experiments could include optical pumping of structures with a very thin or no top metal layer.

\section{CONCLUSION}

We have numerically investigated the possibility to achieve band-edge lasing in optically thick gold-clad PhC membranes. Gold was chosen as cladding metal due to the relatively low absorption loss in the infrared. 2-D eigenmode-expansion calculations were performed for finite 1D-periodic structures. Bandedge lasing was identified both for decoupled modes as for leaky modes. For a membrane thickness of $t=1.0 \mu \mathrm{m}$, a cavity length of 20 periods and 100-nm-thick gold layers, $Q$-factors are of the order of 3000 for the decoupled mode, and 1500 for the leaky mode, and material gain thresholds $G_{\text {th }}$ (for four quantum wells) are about $1200 \mathrm{~cm}^{-1}$ and $2200 \mathrm{~cm}^{-1}$. Emission efficiencies are less than $2 \%$ and about $12 \%$, respectively. For the leaky mode, the emission efficiency can be increased by decreasing the thickness of the patterned gold layer, while increasing the cavity length to preserve the $Q$-factor. A 40 -periods long cavity with a 60-nm-thick patterned gold layer has an emission efficiency of $33.5 \%$ with a $Q$ of 1860 and a $G_{\text {th }}$ of about $1900 \mathrm{~cm}^{-1}$. Both for leaky and uncoupled modes, care has to be taken to avoid coupling with lossy higher order modes.
This can be verified by calculating the band diagram of the used optically thick gold-clad PhC-WG.

We conclude that the optically thick gold-clad $\mathrm{PhC}$ membrane opens up the possibility for electrically pumped surface-emitting band-edge lasing in cavities with a length down to $30 \mu \mathrm{m}$.

\section{REFERENCES}

[1] O. J. Painter, A. Husain, A. Scherer, J. D. O'Brien, I. Kim, and D. Dapkus, "Room temperature photonic crystal defect lasers at near-infrared wavelengths in InGaAsP," J. Lightw. Technol., vol. 17, no. 11, pp. 2082-2088, Nov. 1999.

[2] J. P. Dowling, M. Scalora, M. J. Bloemer, and C. M. Bowden, "The photonic band edge laser: A new approach to gain enhancement," J. Appl. Phys., vol. 75, no. 4, pp. 1896-1899, Feb. 1994.

[3] K. Sakoda, "Low-threshold laser oscillation due to group-velocity anomaly peculiar to two- and three-dimensional photonic crystals," Opt. Express, vol. 4, no. 12, pp. 481-489, Jun. 1999.

[4] S. Nojima, "Optical-gain enhancement in two-dimensional active photonic crystals," J. Appl. Phys., vol. 90, no. 2, pp. 545-551, Jul. 2001.

[5] J. Mouette, C. Seassal, X. Letartre, P. Rojo-Romeo, J.-L. Leclercq, P. Regreny, P. Viktorovitch, E. Jalaguier, P. Perreau, and H. Moriceau, "Very low threshold vertical emitting laser operation in InP graphite photonic crystal slab on silicon," Electron. Lett., vol. 39, no. 6, pp. 526-528, Mar. 2003.

[6] C. Monat, C. Seassal, X. Letartre, P. Regreny, P. Rojo-Romeo, P. Viktorovitch, M. Le Vassor d'Yerville, D. Cassagne, J.-P. Albert, E. Jalaguier, S. Pocas, and B. Aspar, "InP-based two-dimensional photonic crystal on silicon: In-plane bloch mode laser," Appl. Phys. Lett., vol. 81, no. 27, pp. 5102-5104, Dec. 2002.

[7] H.-Y. Ryu, S.-H. Kwon, Y.-J. Lee, Y.-H. Lee, and J.-S. Kim, "Very-lowthreshold photonic band-edge lasers from free-standing triangular photonic crystal slabs," Appl. Phys. Lett., vol. 80, no. 19, pp. 3476-3478, May 2002.

[8] H.-Y. Ryu and M. Notomi, "Finite-difference time-domain investigation of band-edge resonant modes in finite-size two-dimensional photonic crystal slab," Phys. Rev. B, vol. 68, no. 4, p. 045 209, Jul. 2003.

[9] S. Noda and M. Imada, "2d photonic crystal surface-emitting laser using triangular-lattice structure," IEICE Trans. Electron., vol. E85-C, no. 1, pp. 45-51, Jan. 2002.

[10] M. Buda, J. Hay, H. H. Tan, J. Wong-Leung, and C. Jagadish, "Low loss, thin p-clad 980-nm InGaAs semiconductor laser diodes with an asymmetric structure design," IEEE J. Quantum Electron., vol. 39, no. 5, pp. 625-633, May 2003.

[11] P. Bienstman and R. Baets, "Optical modeling of photonic crystals and vcsels using eigenmode expansion and perfectly matched layers," Opt. Quant. Electron., vol. 33, no. 4/5, p. 327, 2001.

[12] CAMFR (Cavity Modeling Framework) [Online]. Available: http:// camfr.sourceforge.net/

[13] K. Sakoda, "Symmetry, degeneracy, and uncoupled modes in two-dimensional photonic lattices," Phys. Rev. B, vol. 52, no. 11, pp. 7982-7986, Sep. 1995.

[14] T.-A. Ma, Z.-M. Li, T. Makino, and M. S. Wartak, "Approximate optical gain formulas for $1.55-\mu \mathrm{m}$ strained quaternary quantum-well lasers," IEEE J. Quant. Electron., vol. 31, no. 1, pp. 29-34, Jan. 1995.

[15] F. Niklaus, P. Enoksson, P. Griss, E. Kälvesten, and G. Stemme, "Lowtemperature wafer-level transfer bonding," J. Microelectromech. Syst., vol. 10, no. 4, pp. 525-531, Dec. 2001.

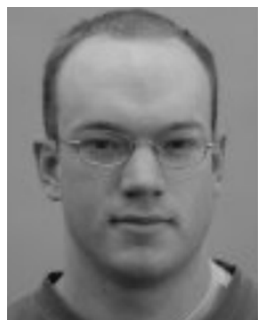

Joris Van Campenhout (S'02) was born in Brussels, Belgium, in 1979. He received the M.Sc. degree in engineering physics from Ghent University, Ghent, Belgium, in 2002. He is currently working towards the $\mathrm{Ph} . \mathrm{D}$. degree in electrical engineering. 


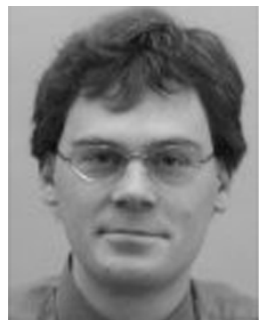

Peter Bienstman (S'97-M'02) was born in Ghent, Belgium, in 1974. He received the M.Sc. degree in electrical engineering and the Ph.D. degree in information technology from Ghent University, Ghent, Belgium, in 1997 and 2001, respectively, where he is currently a Postdoc.

During 2001-2002, he spent the year in the Joannopoulos Research Group, Massachusetts Institute of Technology (MIT), Cambridge. He has published several papers and has one patent. His research interests include the modeling of optical structures, notably photonic crystal structures, vertical-cavity surface-emitting lasers, and resonant-cavity light-emitting diodes.

Dr. Bienstman is a member of IEEE LEOS.

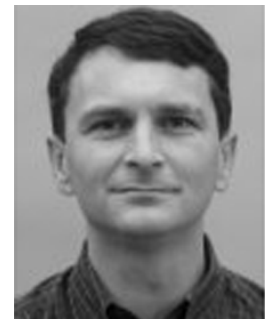

Roel Baets (M'88-SM'96) received the M.Sc. degree in electrical engineering from Ghent University, Ghent, Belgium, in 1980, the M.Sc. degree in electrical engineering from Stanford University, Stanford, CA, in 1981, and the Ph.D. degree from Ghent University in 1984.

$\mathrm{He}$ is the Head of the Optoelectronics Group, Department of Information Technology, Ghent University. Since 1981, he has been with the Department of Information Technology (INTEC), Ghent University, where he is a Professor since 1989. He has worked in the field of III-V devices for optoelectronic systems. With about 200 publications and conference papers, he has made contributions to the design and fabrication of semiconductor laser diodes, passive guided wave devices, PICs, and micro-optic components.

Dr. Baets is a member of the Optical Society of America, IEEE-LEOS, SPIE, and the Flemish Engineers Association. 